Stuart E. Mirvis

Kathirkamanathan

Shanmuganagthan

\section{Imaging hemidiaphragmatic injury}

Received: 1 July 2006

Revised: 10 October 2006

Accepted: 17 November 2006

Published online: 17 February 2007

(C) Springer-Verlag 2007

S. E. Mirvis - K. Shanmuganagthan Department of Diagnostic Radiology and the Maryland Shock-Trauma Center,

University of Maryland

School of Medicine,

22 South Greene Street,

Baltimore, MD 21201, USA

S. E. Mirvis $(\bowtie)$

Department of Radiology,

University of Maryland Medical Center,

22 South Greene Street,

Baltimore, MD 21201, USA

e-mail: smirvis@umm.edu

Tel.: +1-410-3288845

Fax: +1-410-3288925

\begin{abstract}
The supine chest radiograph is the initial and most commonly performed imaging study to evaluate the thorax after trauma. Whenever the chest radiograph is equivocal or suspicious for acute diaphragmatic injury (DI), computed tomography $(\mathrm{CT})$ is usually the next study of choice since it is both generally available and often used to examine other body regions in the patient after trauma. CT is usually diagnostic, particularly if supplemented by multiplanar reformation (MPR) obtained using thin-slice axial scanning and overlapping images for reformations. Magnetic resonance imaging (MRI) is potentially useful to assess the diaphragm if CT findings are indeterminate and the patient is stable enough to have the procedure. Simple T1-weighted spin-echo images in the sagittal and coronal orientation
\end{abstract}

are usually sufficient to establish or exclude DI. This article reviews imaging modalities and strategies for diagnosing DI from blunt trauma.

Keywords Diaphragm injury · Computed tomography $\cdot \mathrm{CT}$. MR imaging

\section{Overview of diaphragm injury (DI)}

The diagnosis of DI is often missed, since distinct clinical and radiologic signs may both be absent or subtle. In most cases, injuries to the diaphragm are not seen in isolation and there are often concurrent injuries involving the solid abdominal organs, pelvis, central nervous system, and mediastinum that may divert attention from DI [1-3]. Detection of DI is often challenging both clinically and by imaging. A careful review of the literature indicates that the majority of DI is caused by penetrating trauma (penetrating: blunt trauma $=2: 1)[3-6]$. DI occurs in $6 \%$ of patients following major blunt force trauma to the lower chest or abdomen and is found in from 3 to $8 \%$ of patients undergoing emergency celiotomy for blunt abdominal trauma [7-16]. The supine chest radiography serves as the initial screening study performed to evaluate the diaphragm, but is, in many centers, being replaced by multi-detector computed tomography (MDCT) survey of the entire torso. Studies based on conventional single-slice CT have reported poor accuracy in diagnosing DI [17, 18]. However, more recent literature indicates that the capacity to obtain high quality axial images with coronal and sagittal MPR using helical or particularly MDCT has improved accuracy [19-23]. 


\section{Mechanism of injury}

There are several postulates of the mechanism of DI in blunt trauma. These include avulsion of the diaphragm attachments, shearing effects from lateral impact to the chest wall, rib fracture fragments penetrating the diaphragm, and sudden transmission of force through the abdominal viscera acting as a hydrodynamic fluid leading to disruption [17]. The dominance of left-sided over rightsided rupture has been attributed to the protection of the right hemidiaphragm by the liver. The liver in close proximity to the right diaphragm prevents transmission of increased intra-abdominal pressure directly to the diaphragm, with the liver acting as a buffer. Experimental studies also suggest that the right hemidiaphragm is mechanically stronger than the left and requires a larger force to disrupt it $[8,17,24]$.

Reiff et al. [25] have shown that DI is more likely to occur $(P<0.0001)$ in motor vehicle impacts that involve significant occupant compartment intrusion $(70.6 \mathrm{~cm}$ versus $48.3 \mathrm{~cm})$ and high velocity changes $(48.8 \mathrm{~km} / \mathrm{h}$ versus $33.9 \mathrm{~km} / \mathrm{h}$ ) compared with impacts without DI. The majority of DI diagnosed preoperatively following blunt trauma are more than $10 \mathrm{~cm}$ in length, but up to $84 \%$ of the diaphragm tears from penetrating injuries are less than $2 \mathrm{~cm}$ in length [24]. A positive pressure gradient of 7$20 \mathrm{~cm}$ of water exists between the peritoneal and pleural spaces, which facilitates herniation of abdominal viscera through a traumatic diaphragm defect [26].

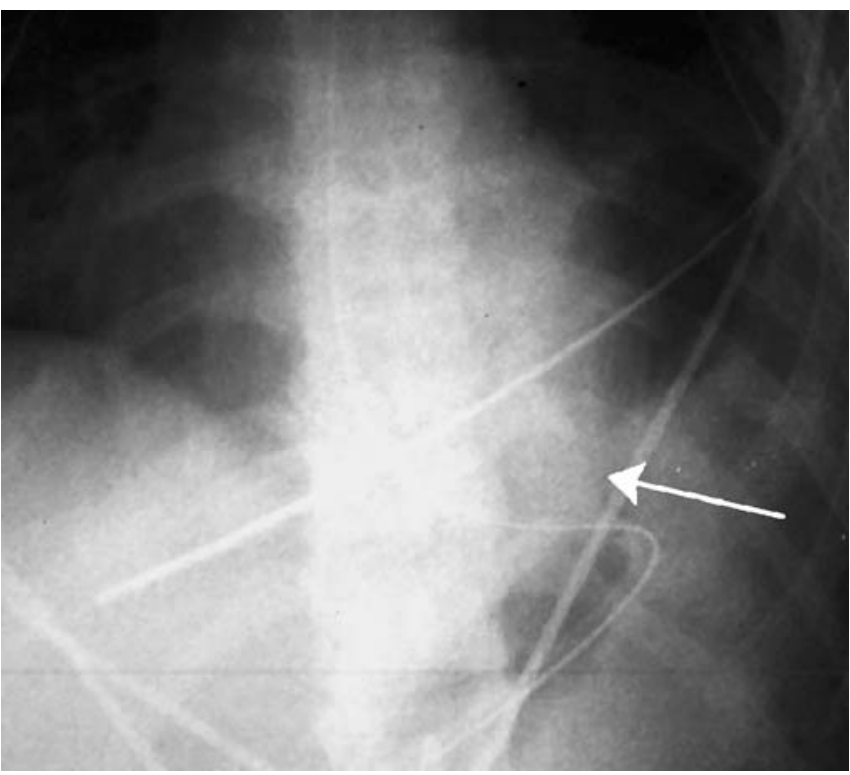

Fig. 1 Collar sign. Anteroposterior radiograph of blunt trauma patient shows a focal constriction in the gastric body (arrow) with fundus projecting into the lower left hemithorax
Injury location

A congenital weakness existing along the embryonic fusion of the costal and lumbar parts of the diaphragm predisposes this site to injury [27, 28]. Blunt left-sided hemidiaphragmatic injuries are usually located at the postero-lateral aspect of the hemidiaphragm between the spleen and the abdominal aorta and extend medially in a radial orientation towards the central tendon. Left-sided DI occurs two to three times more commonly than right-sided injuries following blunt trauma $[8,11,28]$, although some of this difference is related to underdiagnosis of right-sided injuries. An equal incidence of left and right-sided DI is seen from penetrating trauma and among blunt trauma victims who die before reaching definitive medical care [35]. Bilateral hemidiaphragm injuries are uncommon, reported in $2-6 \%$ of patients with DI [4, 15, 24]. Patients with right-sided hemidiaphragm rupture have higher pre-
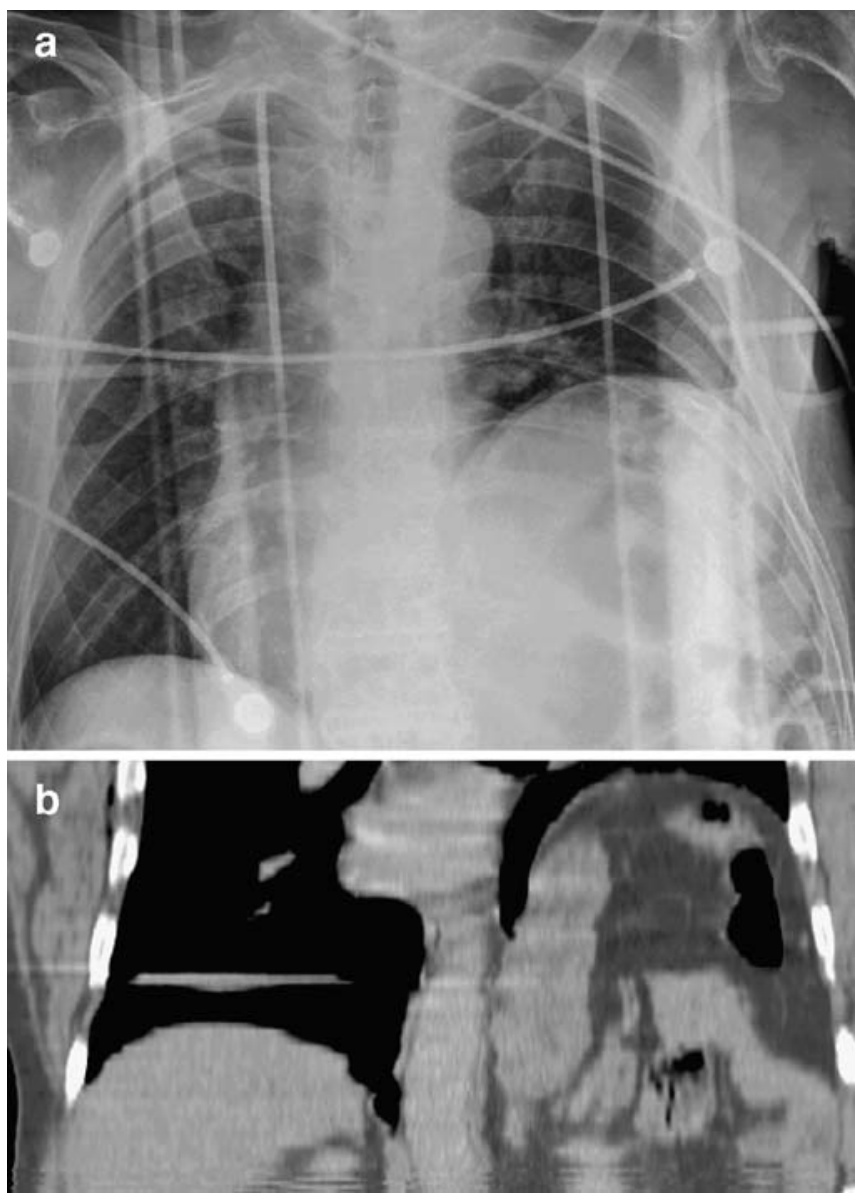

Fig. 2a, b Left hemidiaphragm eventration. a Chest radiograph of blunt trauma patient reveals a markedly elevated, but smoothly contoured apparent left hemidiaphragm. The heart and mediastinum are displaced to the right, but there is no evidence of pleural effusion. b A coronal multiplanar reformatted (MPR) image shows a smooth rounded diaphragm contour without evidence of disruption. The appearance is typical of diaphragm eventration 
hospital mortality resulting from the greater impacting force required to produce a right DI [3, 7, 8, 24, 28, 29]. The apparent low incidence of right-side DI in clinical studies has been attributed to the mass of the liver preventing transmission of high intraabdominal pressure to the right hemidiaphragm, and the subtlety of radiologic signs of right hemidiaphragm rupture, especially without concurrent herniation of abdominal viscera. Experimental studies consistently indicate that greater force is required to rupture the right hemidiaphragm than the left. The stomach and colon are most often herniated on the left and the liver dome on the right.

\section{Associated injuries}

The location of the diaphragm and its proximity to adjacent intrathoracic and intraabdominal organs accounts for associated injuries, which occur in $52-100 \%$ of patients $[1,7,11,15,29,31]$. Intrathoracic injuries such as pneumohemothoraces and multiple rib fractures are seen in $90 \%$ of patients with blunt DI [17, 30, 31]. Meyers et al. [9] reported a 5\% incidence of aortic injury in 68 patients with diaphragm rupture at a level I trauma center. Common associated intraabdominal injuries involve the spleen, in $27-60 \%$ of cases, and liver in $93 \%$ of patients with right and $24 \%$ of patients with left-sided diaphragm ruptures [1, $7,15,16]$. Other common concurrent extrathoracic and extraabdominal injuries include pelvic fractures (40-55\%), long bone fractures $(45-85 \%)$, and closed head injury (25$55 \%)[9,32]$.

\section{Imaging of the DI}

\section{Chest radiography}

Chest radiography is the initial and most commonly performed imaging study to evaluate the diaphragm following trauma, although this practice is declining with increasing use of MDCT screening of the chest after blunt trauma. There are two diagnostic radiographic findings of diaphragmatic rupture with concurrent visceral herniation. The first is herniation of abdominal viscera above the hemidiaphragm into the lower hemithorax in the setting of trauma and/or a focal constriction of the herniated mass at the site of the diaphragm tear, producing circumferential compression ("collar sign") (Fig. 1). Nonspecific chest radiographic findings suggestive of DI on chest radiography include obliteration or distortion of the diaphragm contour, pleural effusion, apparent hemidiaphragm elevation), air-fluid levels in the lower thorax, and contralateral mediastinal shift. Often these nonspecific radiologic signs result from other traumatic and non-traumatic lung base pathology, including atelectasis, effusion, pulmonary contusion, post-trauma pneumatoceles, loculated pneumothorax, aspiration pneumonia, phrenic nerve palsy, segmental or total congenital eventration of the hemidiaphragm (Fig. 2), gastric dilatation, and subphrenic fluid collections. These findings can either mask or mimic signs of DI and visceral herniation.

Initial chest radiographs are normal or nonspecific in $20-50 \%$ of patients with DI. This may account for the great variability (7-66\%) of initially missed DI seen in polytrauma patients reported in the literature $[3,24,30$, $31,33,34]$. Admission radiographs are diagnostic in $27-$ $62 \%$ of patients with left-sided and in $18-33 \%$ of cases with right-sided injuries $[1,17,26,35,36]$. Chest radiographs are suggestive but not diagnostic in another $18 \%$ of cases $[22,33]$. Serial chest radiographs are often useful. As concurrent trauma related acute pulmonary abnormalities in the lower thorax resolve, findings of DI may become more evident on radiographis obtained in the subacute or chronic phase. The diagnosis of DI may be delayed for patients receiving positive pressure ventilatory support, since the natural pressure gradient across the pleuro-peritoneal cavity is abolished or reversed and visceral herniation may not occur until positive pressure ventilator support is terminated $[17,31]$.

Table 1 MDCT protocols

\begin{tabular}{|c|c|c|c|c|c|c|c|c|}
\hline MDCT & $\begin{array}{l}\text { Contrast } \\
\text { volume }\end{array}$ & $1_{2} \mathrm{mg} / \mathrm{ml}$ & Rate & Delay & $\begin{array}{l}\text { Detector } \\
\text { width }\end{array}$ & Pitch & $\begin{array}{l}\text { Rotation } \\
\text { time }\end{array}$ & Bolus pro $^{\mathrm{a}}$ \\
\hline 16-slice & $150 \mathrm{ml}$ & $300 \mathrm{mg} / \mathrm{ml}$ & $\begin{array}{l}90 \mathrm{ml}-6 \mathrm{ml} / \mathrm{s} \\
60 \mathrm{ml}-4 \mathrm{ml} / \mathrm{s}\end{array}$ & Bolus pro $^{a}$ & $0.75 \mathrm{~mm}$ & 0.938 & $0.5 \mathrm{~s}$ & Ascending aorta \\
\hline 40 -slice $<50$ years & $100 \mathrm{ml}$ & $350 \mathrm{mg} / \mathrm{ml} \mathrm{N}$.saline & $\begin{array}{l}50 \mathrm{ml}-6 \mathrm{ml} / \mathrm{s} \\
50 \mathrm{ml}-4 \mathrm{ml} / \mathrm{s} \\
50 \mathrm{ml}-5 \mathrm{ml} / \mathrm{s}\end{array}$ & $18 \mathrm{~s}$ & $0.625 \mathrm{~mm}$ & 0.976 & $0.5 \mathrm{~s}$ & Ascending aorta \\
\hline 40 -slice $>50$ years & $100 \mathrm{ml}$ & $350 \mathrm{mg} / \mathrm{ml} \mathrm{N}$.saline & $\begin{array}{l}50 \mathrm{ml}-6 \mathrm{ml} / \mathrm{s} \\
50 \mathrm{ml}-4 \mathrm{ml} / \mathrm{s} \\
50 \mathrm{ml}-5 \mathrm{ml} / \mathrm{s}\end{array}$ & Bolus pro $^{a}$ & $0.625 \mathrm{~mm}$ & 0.976 & $0.5 \mathrm{~s}$ & Ascending aorta \\
\hline
\end{tabular}

\footnotetext{
${ }^{\mathrm{a}}$ Threshold, $90 \mathrm{Hu}$
} 
CT

\section{Technique}

Conventional CT has a variable sensitivity of $14-61 \%$ and specificity of $76-99 \%$ for diagnosing diaphragm rupture $[6,13,32,33,35]$. Limitations of conventional CT include difficulty in visualizing the entire dome-shaped diaphragm on axially oriented images, low-resolution sagittal and coronal reformatted images performed with $8-10 \mathrm{~mm}$ axial slice thickness, and difficulty differentiating the diaphragm
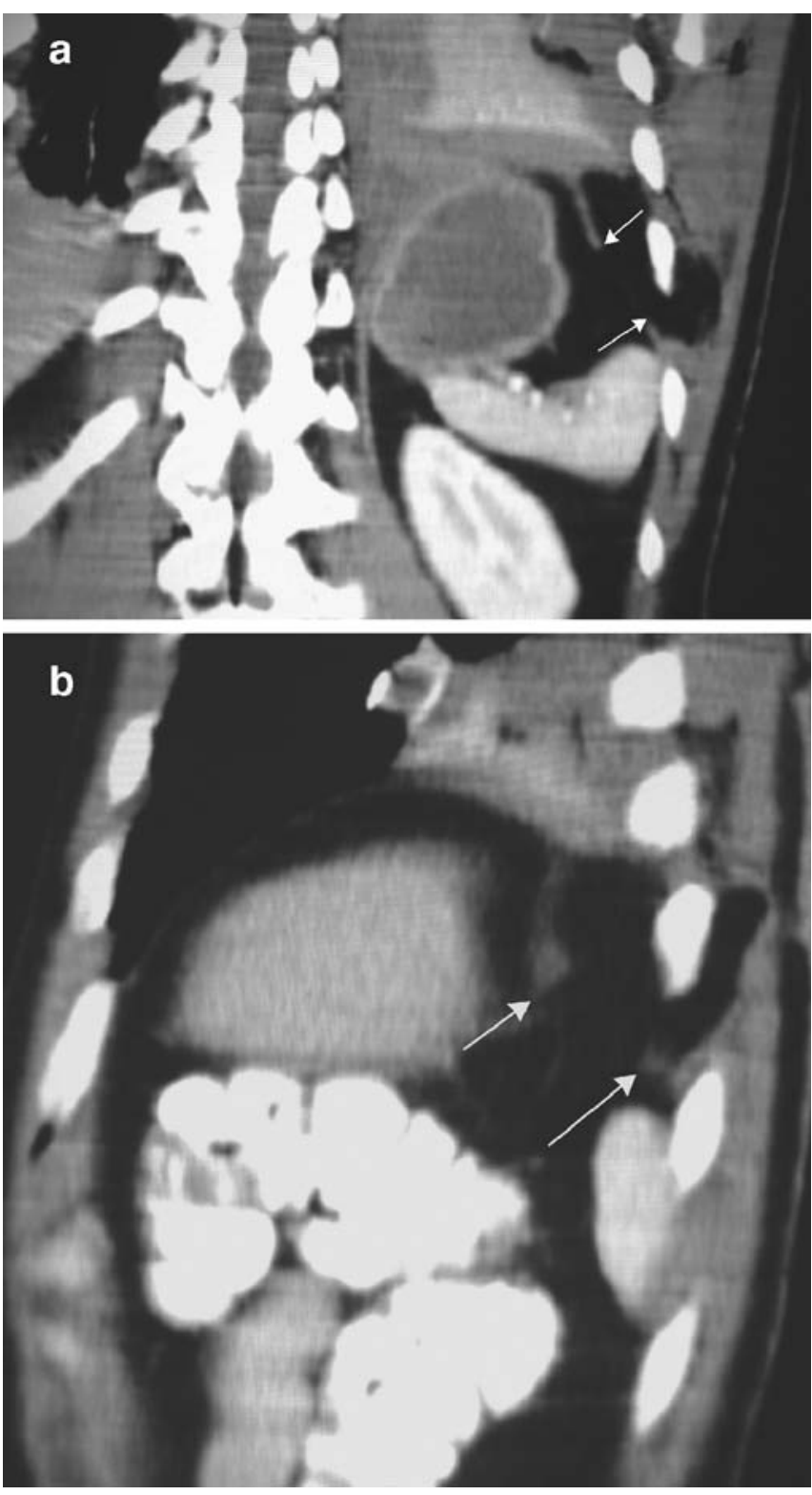

Fig. 3a, b CT of a direct diaphragm disruption. a Coronal MPR image in blunt trauma victim shows direct discontinuity in the left hemidiaphragm (arrows) with herniation of abdominal fat into the thoracic cavity. Also note extension of fat through the chest wall. b Sagittal MPR of same injury from adjacent pulmonary pathology or normal soft tissue structures. Spiral CT, particularly with multiple detectors, can overcome these limitations through use of thin-slice collimation and slice overlap to optimize $\mathrm{z}$-axis resolution, the capacity to obtain large volumes of data during a single breath hold, and decreased motion and misregistration artifacts $[37,38]$. The authors currently use MDCT-16 and MDCT-40 to study most acute trauma patients using the "whole body" protocol shown in Table 1. Currently, many commercial computer systems are available to render images in multiple planes using various techniques. Reconstructed images are routinely obtained in patients with chest radiographic findings suspicious for DI, abnormally elevated hemidiaphragms on axial MDCT, and major injuries to the upper abdomen or lower chest. Using these data, three-dimensional (3D), sagittal and coronal MPR images are obtained at 2-mm intervals. This technique yields high spatial resolution axial, MPR, and 3D images to assess diaphragm contour and its relationship to the abdominal viscera $[38,39]$. Patients presenting with suspicious findings for DI by chest radiography in the subacute or chronic posttrauma period, are scanned using the same collimation and pitch following administration of intravenous contrast material.

\section{Blunt trauma}

Since blunt DI is highly associated with torso injuries, the majority of hemodynamically stable patients with blunt DI will require a CT examination soon after admission to evaluate sites and extent of coexisting thoraco-abdominal injuries. Diagnostic CT signs of diaphragm injury include a defect in the continuity of the diaphragm or crus (Fig. 3) and a waist-like constriction of abdominal viscera or omentum at the site of herniation as seen on axial or reformatted CT images, the CT "collar sign" (Figs. 4, 5). Other CT signs include herniation of abdominal viscera or omentum into the thoracic cavity through a diaphragm disruption (Figs. 3, 4, 5, 6), and thickening of the diaphragm as a result of edema or hematoma.

Studies using conventional CT report that direct visualization of a diaphragm defect is the most sensitive CT finding in $71-73 \%$ of patients with diaphragm rupture [40]. The CT "collar sign" has been reported as $100 \%$ specific, but with a limited sensitivity of $27-36 \%$ for diagnosing DI $[33,40]$. The authors performed a retrospective study [19] to determine sensitivity and specificity of single-slice spiral $\mathrm{CT}$ in diagnosing blunt diaphragm injury. Spiral CT was performed on 41 patients ( 32 had left hemidiaphragm injuries, ten right and one patient with bilateral hemidiaphragm injury) with chest radiographic findings suspicious for DI. Unlike previous studies, in this study the most common CT finding of diaphragmatic rupture was the CT "collar sign" with a sensitivity of $63 \%$ and specificity of $100 \%$. A defect in the crus or diaphragm was only $22 \%$ 

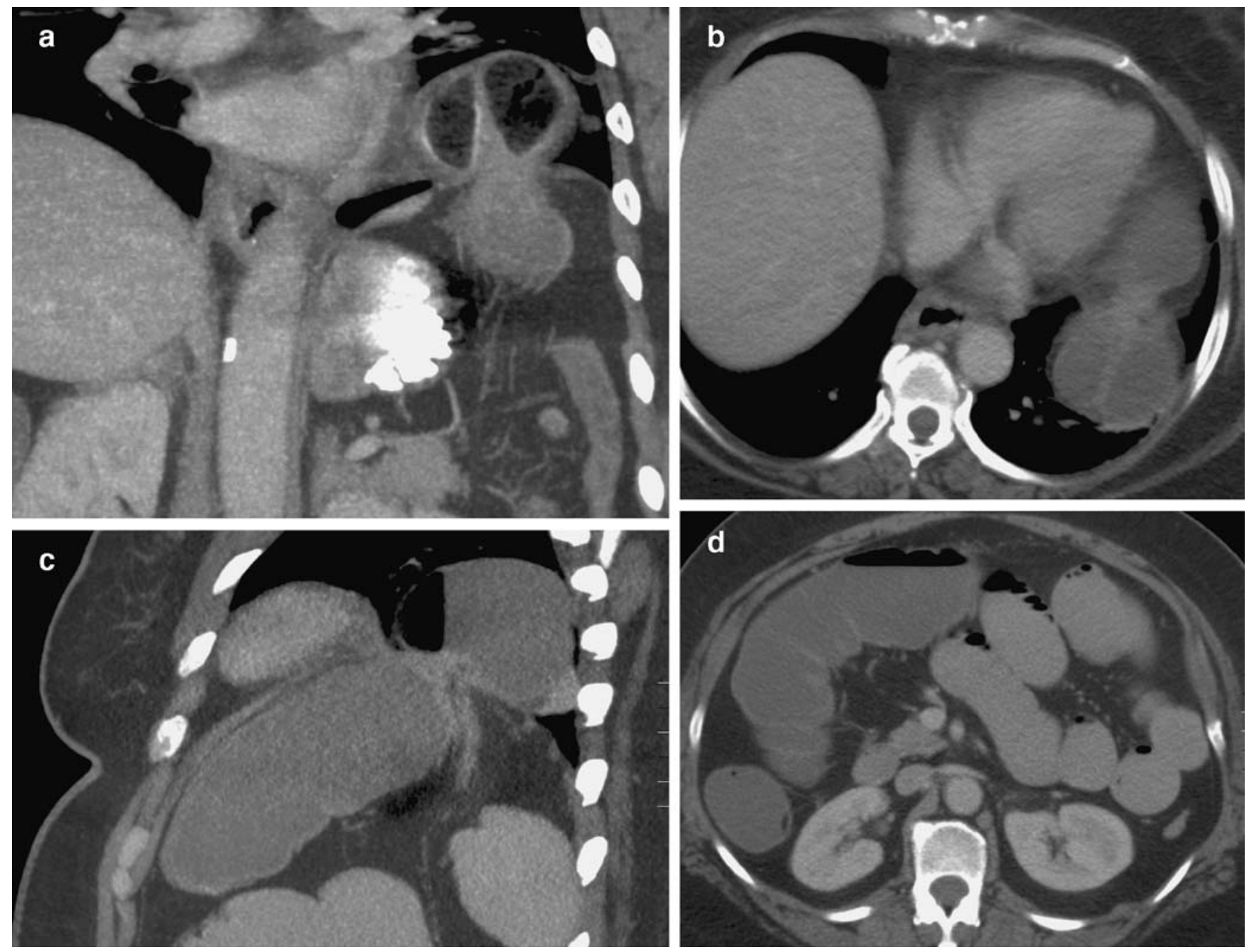

Fig. 4a-d Small bowel herniation after straining for bowel movement. a The patient developed nausea and vomiting a few hours after straining at bowel movement. The CT image shows small bowel loop herniated across left hermidiaphragm with collar sign at level of tear. Fluid distends herniated bowel loop. b Coronal and c sagittal MPRs show obstructed herniated small bowel with collar sign. Note on sagittal MPR contact of bowel loop with posterior thoracic wall. d Axial image confirms small bowel obstruction sensitive to detect left-sided DI [19]. Reformatted coronal and sagittal images were especially helpful for detection of subtle right-sided visceral herniation and to delineate the diaphragm outline. Reformatted spiral CT was useful to exclude the diagnosis in 17 patients with chest radiographic findings suspicious for acute DI. Overall, single-slice spiral CT was $78 \%$ sensitive for left and $50 \%$ sensitive for right hemidiaphragm rupture and was $100 \%$ specific for both right and left-sided injuries. Spiral CT failed to diagnose DI when there was no associated visceral herniation.

Another CT sign that is useful for diagnosing rupture of the diaphragm is the "dependent viscera sign" (Figs. 4, 5, 6, 7). Normally, the abdominal viscera are held off the posterior chest wall by the support of the intact hemidiaphragms. If there is a tear in the diaphragm with herniation of the viscera into the thorax the visceral will fall directly onto the posterior chest wall in the supine patient. The dependent viscera sign is present when the upper onethird of the liver abuts the posterior ribs on the right or if the stomach, spleen, or bowel abut the left posterior ribs. In this way, rupture with visceral herniation can be diagnosed from axial CT images without directly imaging the diaphragm rent or edges of the tear. This sign was initially described by Bergin et al. [22] in ten patients. The dependent viscera sign was seen on CT in $100 \%$ of patients with left-sided and $83 \%$ of patients with right-sided rupture. Detection of diaphragmatic rupture was $90 \%$ using the dependent viscera sign with complete interobserver agreement [22]. A study performed by Nchimi et al. [42] assessed CT findings in 179 blunt trauma patients, including 11 with left-sided and five with right-sided diaphragm rupture. The presence or absence of 11 
Fig. 5a, b CT collar sign. a Coronal volume-rendered image shows distinct collar sign (arrow). b Sagittal volume-rendered image shows collar sign and contact of the stomach directly with the posterior chest wall (dependent viscera sign)
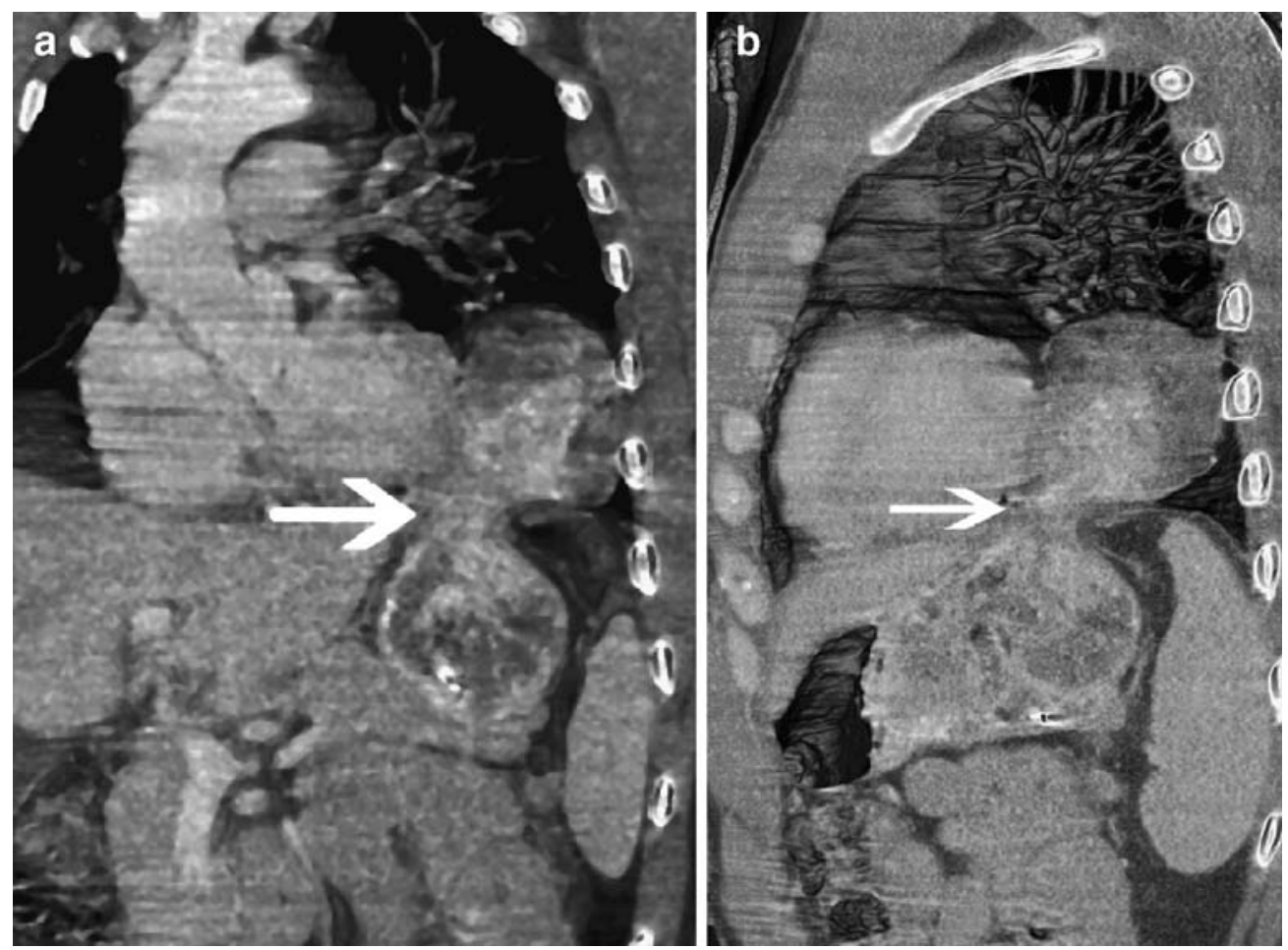

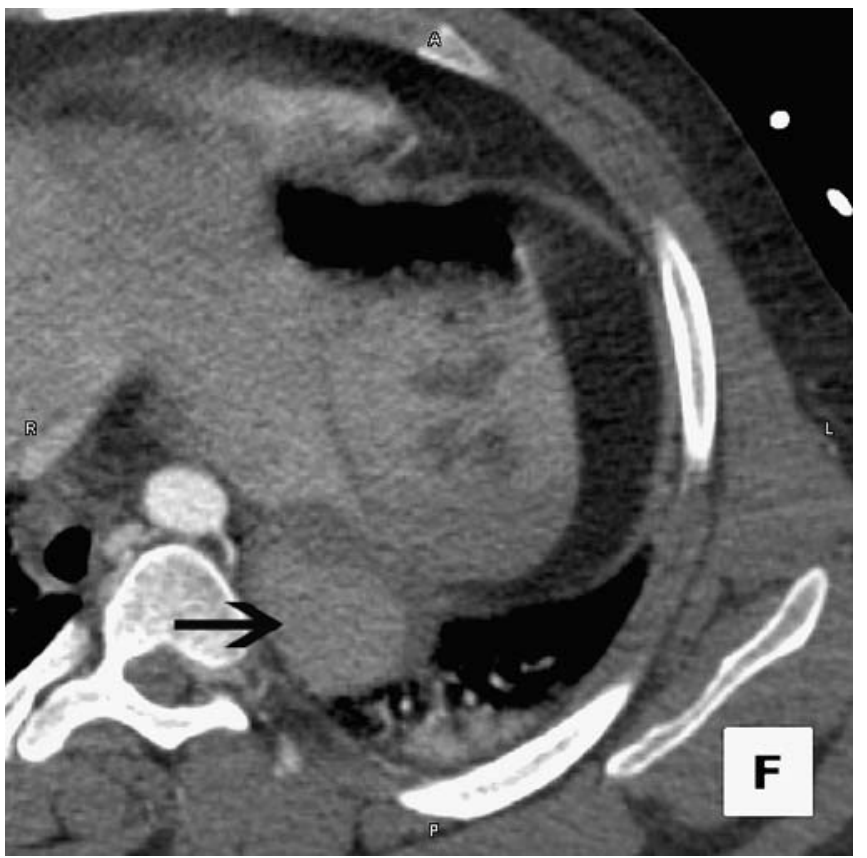

Fig. 6 Dependent viscera sign. CT image of trauma patient shows a soft tissue mass in the posterior medial left inferior thoracic cavity in direct contact with posterior chest wall (arrow). The medial left hemidiaphragm is interrupted by the mass. At surgery the superior pole of the spleen was herniated through a traumatic posterior medial diaphragm defect previously reported $\mathrm{CT}$ signs of rupture were tabulated. Strong predictors of diaphragm rupture $(P<0.001)$ included (1) direct discontinuity, (2) segmental non-recognition of the diaphragm, (3) intrathoracic visceral herniation, (4) elevation of the hemidiaphragm, and (5) combined hemothorax and hemoperitoneum. A combination of the first three signs was $100 \%$ sensitive [41].

Right-sided blunt DI In a study focusing on right-sided blunt DI conducted at our trauma center, the authors found variants of the collar sign, the "hump" sign and "band" sign (Figs. 8, 9), were also useful for diagnosis. The axial nature of CT makes diagnosis of diaphragm rupture difficult along the horizontal portion. MPR in the coronal and sagittal plane are often degraded by motion. Initially, in our practice, CT of the lower chest and abdomen appeared of limited value due to patient and diaphragm motion, volume averaging, and poor z-axis resolution. The advent of spiral CT and particularly MDCT has improved accuracy for both left- and right-sided injury. The use of thin slice profile $(2.5 \mathrm{~mm}$ MDCT- 4 or $0.75 \mathrm{~mm}$ MDCT16), pitch of 0.75 or 0.625 and $1-2 \mathrm{~mm}$ MPR in the coronal and sagittal plane optimize injury detection. Major CT findings include diaphragm discontinuity, extension of abdominal contents above the diaphragm, a "collar sign" (bowel constriction at the point of the herniation), and the dependent viscera sign. Most right-sided DI is associated with chest and abdominal visceral injuries.

A retrospective study was performed by Rees and Mirvis [20] involving 12 patients with surgically verified 
Fig. 7a-d CT of direct left diaphragm tear and dependent viscera sign. Axial CT images $(\mathbf{a}, \mathbf{b})$ show elevation of the colon (black curved arrows) and mesentery (curved white arrow) and direct defect in the diaphragm (straight white arrows). c Sagittal and d coronal reformations show extent of colon herniation and mesentery and also indicate tear in diaphragm (straight black arrow). Contact of the colon and mesentery with the posterior chest wall constitutes the dependent viscera sign
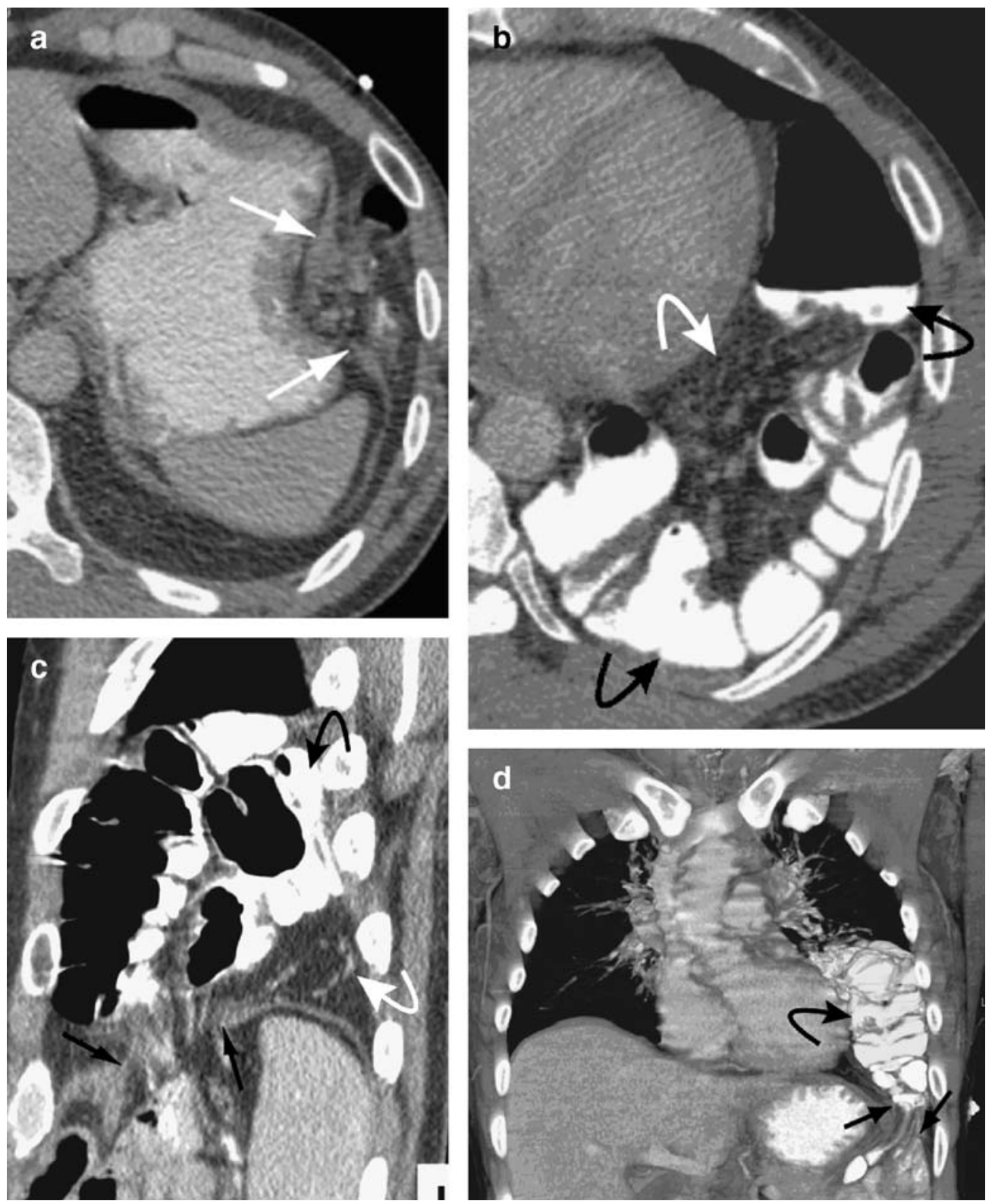

right diaphragm rupture studied by MDCT (four- or 16slice) prior to surgery. Sagittal and coronal MPR was performed in all cases. Studies were analyzed retrospectively for signs of right DI, including direct diaphragm discontinuity, the "collar sign", the "dependent viscera sign", and intra-thoracic location of herniated abdominal contents. Among the 12 patients, direct diaphragm discontinuity was seen in seven $(58 \%)$ cases, the collar sign in five $(42 \%)$, the dependent viscera sign in four $(33 \%)$, and transdiaphragmatic herniation of the right colon and fat in another. Two variants of the collar sign were identified on high-quality sagittal and coronal MPR. The first, termed the "hump sign", is a rounded portion of liver herniating through the diaphragm forming a humpshaped mass, and the second, termed the "band sign", is a linear lucency across the liver along the torn edges of the hemidiaphragm. The hump sign occurred in ten (83\%) and the band sign in four $(33 \%)$ patients. In this review, MDCT appeared very useful and superior to single detector helical CT in the diagnosis of right hemidiaphragm injury, particularly using sagittal and coronal MPR images.

Intrapericardial herniation Intrapericardial herniation of abdominal viscera is a rare occurrence with only 82 cases described in the literature up to 1999 [42, 43]. Among 60 
Fig. 8a-c Hump and band sign of right hemidiaphragm rupture. a Axial CT shows a double liver dome with a low attenuation band in-between (arrow).

b Coronal and c sagittal MPRs show a hump of liver parenchyma (star) protruding above the right hemidiaphragm with indentation of liver (arrow in b) at edge of torn diaphragm. The double contour noted in $\mathbf{a}$ is due to axial slice crossing the herniated right lobe and subdiaphragmatic left lobe with the constricted liver parenchyma (low-attenuation line) in between (arrow)
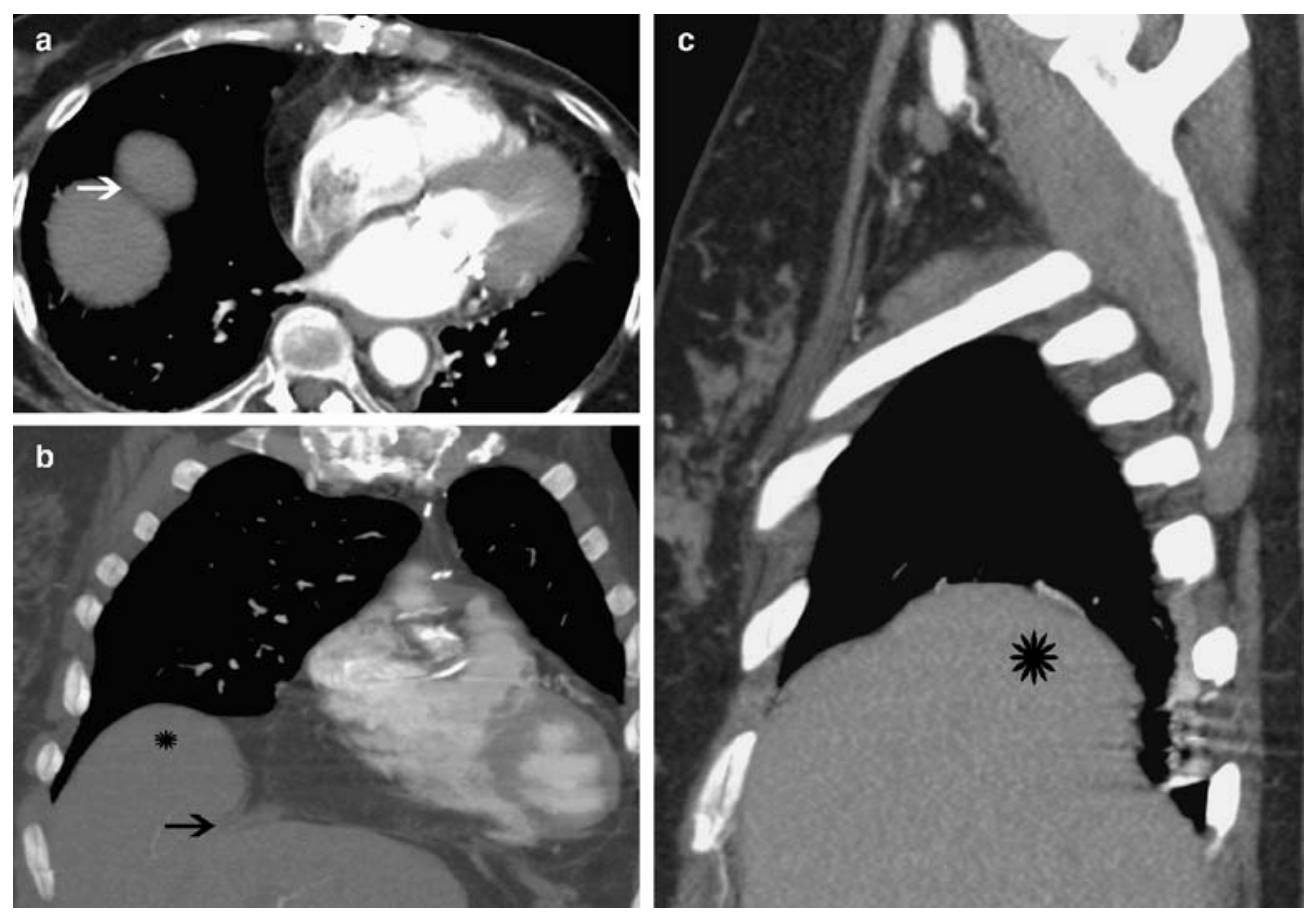

patients sustaining blunt chest trauma described over a 9year period at one trauma center, only two cases involved rupture through the central tendon into the pericardium. As might be expected, other major chest and abdominal injuries typically occur with this lesion. Patients may develop intrapericardial herniation acutely or subacutely after the traumatic event or even years following the trauma. Clinical signs may be related either to bowel obstruction, (usually colon or stomach) or acute/chronic cardiac tamponade. Simultaneous disruption of the pericardium and central tendon may permit cardiac luxation into the peritoneal cavity. The diagnosis can be suggested by radiographs showing gas shadows superimposed over the cardiac shadow, although this would more classically suggest typical left hemidiaphragm rupture and herniation of gas-containing bowel into the chest. The diagnosis can be confirmed by a gastrointestinal contrast study or CT showing abdominal contents confined to the pericardial sac and displacing the heart.

\section{Limitations of $C T$}

In the authors' experience, single-slice spiral CT is limited in its ability to diagnose DI without associated herniation of intraabdominal viscera. Use of relatively thick sections (5 $\mathrm{mm}$ or more) and motion-related artifacts significantly degrade quality of reformations obtained from single-slice systems. No DI that occurred without associated visceral herniation was diagnosed in the authors' retrospective study performed using single-detector spiral CT [19]. The use of axial CT only limits detection of DI since the axial plane is tangential to the diaphragm dome. The higher the quality of coronal and sagittal reformations, as generated by thin and overlapping axial images that are rapidly acquired, the more useful these views will be toward establishing or refuting the diagnosis. In the acute setting, other factors can confound the diagnosis of DI, including concurrent pulmonary pathology related to trauma and left lower lobe atelectasis that can significantly elevate and obscure the left hemidiaphragm. Localized defects occurring mainly in the posterolateral aspect of the left hemidiaphragm can be seen as a normal variant in up to $6 \%$ of asymptomatic adults, especially in elderly females $[23,40,44,45]$. The diagnosis of diaphragm rupture should not be made exclusively based on CT findings of a "localized defect" in the crus or thickening of the diaphragm [46]. Injury to organs adjacent to the diaphragm, such as the adrenal gland, kidney, liver, spleen or proximal small bowel, can produce tracking of hemorrhage or edema along the diaphragm, suggesting primary diaphragm injury.

Confusion in the diagnosis of DI may also result from eventration and some congenital or acquired hernias. Eventration of the diaphragm is an abnormal diaphragm elevation at a site of weakness that may be caused by failure of muscle migration into the diaphragm or weakness of any of the embryonic diaphragmatic components. Eventration more commonly occurs on the left and more often involves the posterior hemidiaphragm. The elevated diaphragm is smoothly contoured and abdominal contents are confined below the thin, but intact hemidiaphragm (Fig. 2). A foramen of Bochdalek hernia is caused by a failure of closure of the pleuroperitoneal hiatus that allows 

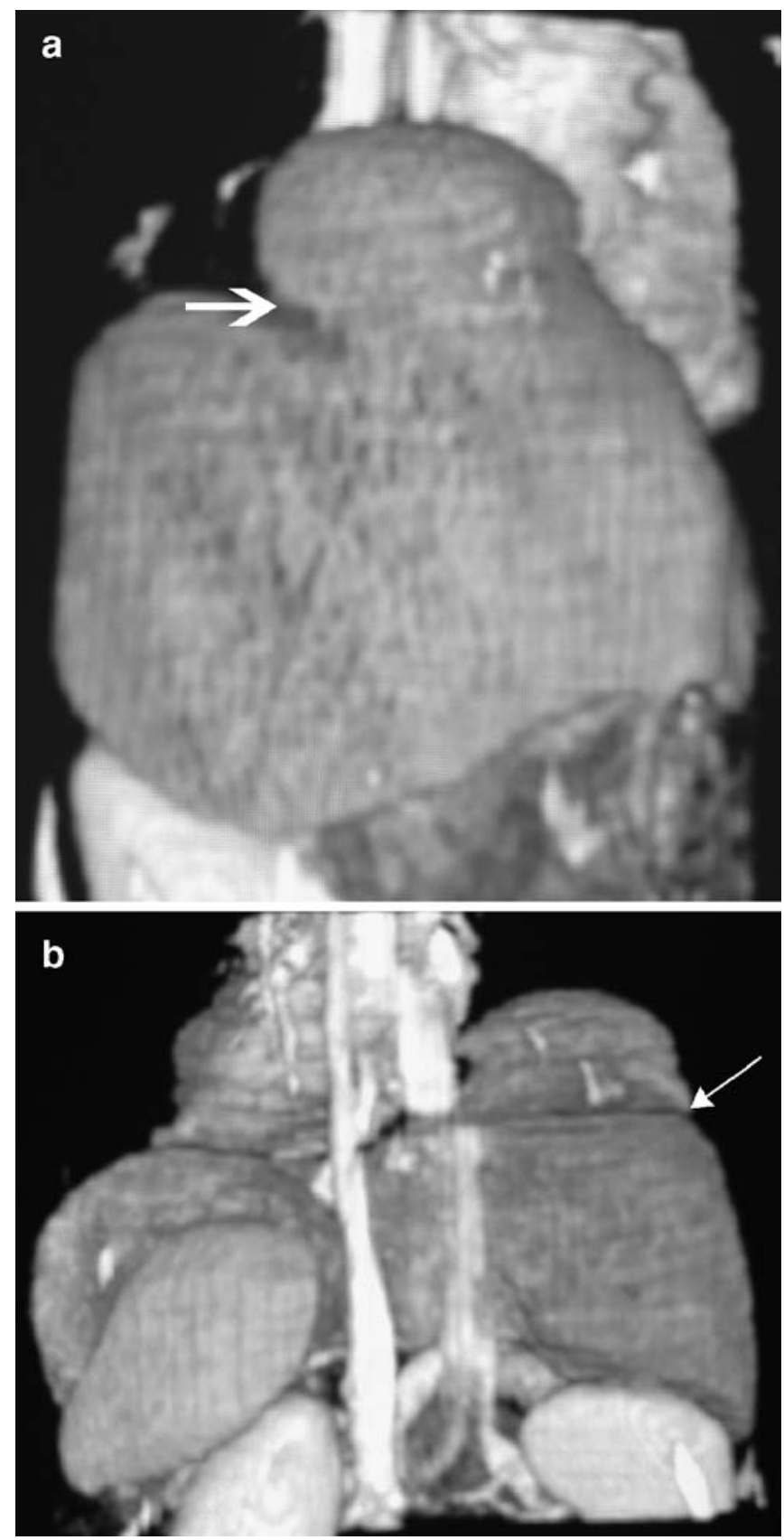

Fig. 9a, b Right hemidiaphragm rupture with hump and band sign a Right lateral and $\mathbf{b}$ posterior perspective volume rendered CT images in blunt trauma patient show hump-shaped liver tissue projecting above plane of diaphragm with low attenuation band across base of hump (arrows) at level of diaphragm tear

protrusion of abdominal viscera into the chest. This abnormality could potentially be diagnosed as a traumatic rupture in the appropriate clinical setting. The Bochdalek hernia is five times more common on the left side than on the right, is rarely seen in adults, but it is the most common form of hernia in infants.

\section{$M D C T$}

More recent studies utilizing MDCT (four and 16 detectors) indicate that this technology is substantially better than single-slice helical CT for establishing the presence of DI [20, 21, 47]. The authors' experience is that coronal and sagittal reformatted images are of major benefit in the CT assessment of the hemidiaphragms in making a diagnosis of diaphragm tear and for increasing confidence in establishing or excluding the diagnosis. Our studies using MDCT for diagnosing blunt traumatic right DI, described above, have been especially convincing.

Magnetic resonance imaging (MRI)

Routine use of respiratory and cardiac gating with direct coronal and sagittal MRI helps to optimally visualize the entire diaphragm [26, 48]. T1-weighted and gradient-echo sequences display the normal diaphragm as a continuous hypointense band. Injuries to the diaphragm are seen as

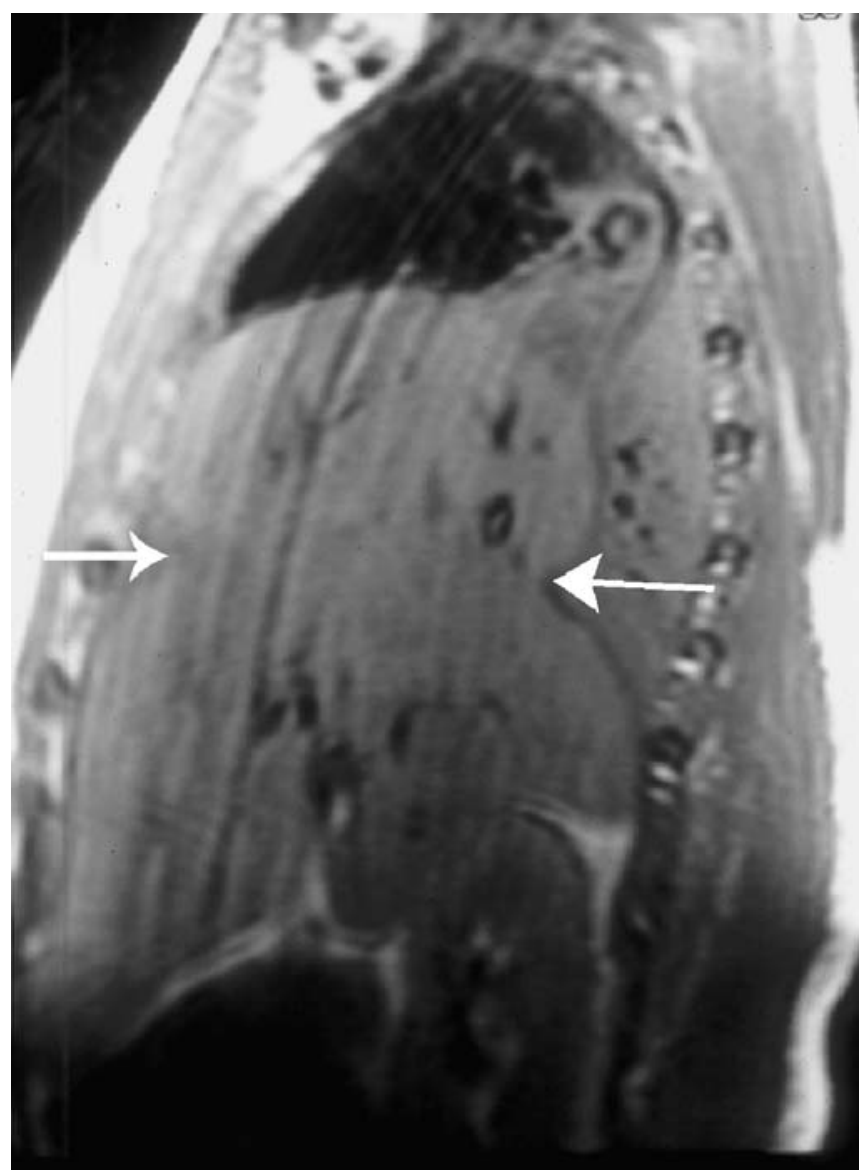

Fig. 10 MRI of right diaphragm rupture. Sagittal MRI through the right hemidiaphragm in blunt trauma victim shows herniation of the right lobe of the lever above the level of the diaphragm with focal indentations in parenchyma at torn edges (arrows). Right lower lobe atelectasis is posterior to herniated liver 
abrupt defect(s) in the low signal intensity hemidiaphragm. Herniation of abdominal viscera or omentum through a diaphragm rupture can be accurately demonstrated by MRI (Fig. 10) [48]. Currently, at our trauma center, the authors use T1-weighted sagittal and coronal images to evaluate the diaphragm in patients presenting with a clinical or radiologic suspicion of DI when the diagnosis is uncertain after MDCT with reformations. While gradient-echo sequences are fast and decrease motion, chemical shift artifact confuses to the analysis of the diaphragm. Singleshot fast spine-echo with a short echo, half-Fourier acquisition, and fast gradient-echo with injection of gadolinium and fat suppression may also be appropriate for diaphragm imaging by decreasing motion and allowing enhancement of atelectasis and pulmonary contusion [23].

\section{Conclusion}

Multiple imaging modalities are available for the preoperative diagnosis of DI. Chest radiographs are the initial and most commonly performed imaging study to evaluate the diaphragm following trauma. When chest radiography is indeterminate spiral CT, preferably with multiple detector rows, is the most appropriate secondary study since the majority of hemodynamically stable patients with blunt DI will require admission $\mathrm{CT}$. Thin, overlapping sections and MPR maximize CT accuracy and should be performed when DI is suspected prior to admission CT. Multidetector spiral CT can be anticipated to improve further on the accuracy of CT to assess for DI, particularly for right-sided injuries. When CT results remain equivocal, T1-weighted sagittal and coronal MRI has proven very useful for both right and left-sided injury.

\section{References}

1. Rodriguez-Morales, Rodriguez A, Shatney CH (1986) Acute rupture of the diaphragm in acute trauma: analysis of 60 patients. J Trauma 26:438-444

2. Grage TB, MacLean LD, Campbell GS (1959) Traumatic rupture of the diaphragm. Surgery 46:669-672

3. Estrera AS, Landay MJ, McCelland RN (1985) Blunt traumatic rupture of the right hemidiaphragm: experience in 12 patients. Ann Thoracic Surg 39:525-530

4. Asensio JA, Demetriades D, Rodriguez A (1992) Injury to the diaphragm. 461-485

5. Calhoon JH, Grover FL, Trinkle JK (1992) Chest trauma approach and management. Clin Chest Med 13:55-66

6. Chen JC, Wilson SE (1991) Diaphragmatic injuries: recognition and management in sixty-two patients. Am Surg 57:810-815

7. Boulanger BR, Milzman DP, Rosati C, Rodriguez A (1993) A comparison of right and left blunt traumatic diaphragmatic rupture. J Trauma 35:255-260

8. Kerney PA, Rouhhana SW, Burney RE (1989) Blunt rupture of the diaphragm: mechanism, diagnosis, and treatment. Ann Emerg Med 18:1326-1330

9. Meyers, McCabe CJ (1993) Traumatic diaphragm hernia: occult marker of serious injury. Ann Surg 218:783-790

10. McHugh K, Ogilvie BC, Brunton FJ (1991) Delayed presentation of traumatic diaphragmatic hernia. Clin Radiol 43:246-250
11. Morgan AS, Flancbaum L, Esposito T et al (1986) Blunt injury to the diaphragm: an analysis of 44 patients. J Trauma 26:565-568

12. Brandt ML, Luks FI, Spigland NA et al (1992) Diaphragmatic injury in children. J Trauma 32:298-301

13. Voller GR, Reisser JR, Fabian TC et al (1990) Blunt diaphragm injury: a five year experience. Am Surg 56:28-31

14. Shah R, Sabanathan S, Mearns AJ et al (1995) Traumatic rupture of the diaphragm. Ann Thorac Surg 60:1444-1449

15. Ward RE, Flynn TC, Clark WP (1981) Diaphragm disruption secondary to blunt abdominal trauma. J Trauma 21:35-38

16. Drew JA, Mercer EC, Benfield JR (1973) Acute diaphragm injury. Ann Thorac Surg 16:67-78

17. Gelman R, Mirvis SE, Gens D (1991) Diaphragmatic rupture due to blunt trauma: sensitivity of plain chest radiographs. AJR Am J Roentgenol 156:51-57

18. Demos TC, Solomon C, Posniak HV, Flisak MJ (1989) Computed tomography in traumatic defects of the diaphragm. Clin Imaging 13:62-67

19. Killeen KL, Mirvis SE, Shanmuganathan K (1999) Helical CT of traumatic diaphragmatic rupture secondary to blunt trauma. AJR Am J Roentgenol 173:1611-1616

20. Rees O, Mirvis SE, Shanmuganathan K (2005) Multidetector-row CT of right hemidiaphragmatic rupture caused by blunt trauma: a review of 12 cases. Clin Radiol 60:1280-1289
21. Tresallet $\mathrm{C}$, Menegaux F, Izzillo R, Nguyen-Thanh Q, Cardot V, Chigot JP (2004) Usefulness of CT reconstructed pictures for diaphragmatic rupture after blunt trauma. J Am Coll Surg 198:666-667

22. Bergin D, Ennis R, Keogh C et al (2001) The "dependent viscera" sign in CT diagnosis of blunt traumatic diaphragmatic rupture. AJR Am J Roentgenol 177:1137-1140

23. Iochum $\mathrm{S}$, Ludig $\mathrm{T}$, Walter $\mathrm{F}$ et al (2002) Imaging of diaphragm injury: a diagnostic challenge? Radiographics 22:S103-S118

24. Wise L, Connors J, Hwang YH et al (1973) Traumatic injuries to the diaphragm. J Trauma 13:946-950

25. Reiff DA, Davis RP, Maclennan PA, McGwin G Jr, Clements R, Rue LW 3rd (2004) The association between body mass index and diaphragm injury among motor vehicle collision occupants. J Trauma 57:1324-1328

26. Marchand P (1957) A study of the forces productive of gastroesophageal regurgitation and herniation through the diaphragmatic hiatus. Thorax 12:189-202

27. Lucido JL, Wall CA (1963) Rupture of the diaphragm due to blunt trauma. Arch Surg 86:989-994

28. Ward RE, Flynn TC, Clark WP (1981) Diaphragmatic disruption secondary to blunt abdominal trauma. J Trauma 21:35-38

29. Epstein LI, Lempke RE (1968) Rupture of the right hemidiaphragm due to blunt trauma. J Trauma 8:19-27 
30. Bekassy SM, Dave KS, Wooler GH et al (1973) "Spontaneous" and traumatic rupture of the diaphragm: long-term results. Ann Surg 177:320-324

31. Beal SL, Mackennon M (1988) Blunt diaphragm rupture: a morbid injury. Arch Surg 123:828-832

32. Shanmuganathan K, Killeen K, Mirvis SE, White CS (2000) Imaging of diaphragmatic injuries. J Thorac Imaging 15:104-111

33. Ilgenfritz FM, Stewart DE (1992) Blunt trauma of the diaphragm: a 15 county private hospital experience. Am Surg 56:334-338

34. Wienecek RG, Wilson RF, Steiger Z (1986) Acute injuries of the diaphragm an analysis of 165 cases. J Thorac Cardio Vasc Surg 92:989-993

35. Shapiro MJ, Heiberg E, Durham RM et al (1995) The unreliability of CT scans and initial chest radiographs in evaluating blunt trauma induced diaphragmatic rupture. Clin Radiol 51:27-30
36. Brant ML, Luks FI, Sipland NA et al (1992) Diaphragmatic injury in children. J Trauma 181:682-686

37. Pagliarello G, Carter J (1992) Traumatic injuries of the diaphragm: timely diagnosis and treatment. J Trauma 33:194-197

38. Israel S, McDaniel PA, Primac SL et al (1996) Diagnosis of diaphragmatic trauma with helical CT in a swine model. AJR Am J Roentgenol 167:637-641

39. Shanmuganathan K, Mirvis SE (1999) Imaging diagnosis of nonaortic thoracic injury. Radiol Clin North Am 37:533-551

40. Glasser DL, Shanmuganathan K, Mirvis SE (1998) General case of the day. Radiographics 18:799-801

41. Worthy SA, Kang EY, Hartman TE et al (1995) Diaphragmatic rupture: CT findings in 11 patients. Radiology 194:885-888

42. Nchimi A, Szapiro D, Ghaye B, Willems V, Khamis J, Haquet L, Noukoua C, Dondelinger RF (2005) Helical CT of blunt diaphragmatic rupture. AJR Am J Roentgenol 184:24-30
43. Reina A, Vidana E, Soriano $P$ et al (2001) Traumatic intrapericardial diaphragmatic hernia: case report and literature review. Injury 32:153-156

44. Katsumura Y, Satoh S (1999) Constriction of liver tissue herniated through a diaphragmatic defect, simulating a pulmonary tumor. Nihon Kokyuki Gakkai Zasshi 37:715-717

45. Gale ME (1985) Bockdalek hernia: prevalence and CT characteristics. Radiology 156:449-452

46. Leung JCM, Nance ML, Schwab CW et al (1999) Thickening of the diaphragm: a new computed tomography sign of diaphragm injury. J Thorac Imaging 14:126-129

47. Mirvis SE (2005) Imaging of acute thoracic injury: the advent of MDCT screening. Semin Ultrasound CT MR 26:305-331

48. Shanmuganatha K, Mirvis SE, White CS et al (1996) MR imaging evaluation of hemidiaphragms in acute blunt trauma: experience with 16 patients. AJR Am J Roentgenol 167:397-402 